\title{
Angioplastia facilitada con dosis completa de tenecteplasa en el infarto agudo de miocardio con elevación del segmento ST
}

En los pacientes con infarto agudo de miocardio con elevación de ST, el empleo de fibrinólisis facilitada con dosis completa de tenecteplasa previo a la realización de un procedimiento percutáneo en las siguientes 3 horas, en comparación con la angioplastia primaria, se asocia a una peor evolución.

In patients with acute myocardial infarction with ST-segment elevation facilitated angioplasty with full dose of tenecteplase, compared with primary angioplasty, is associated with an increase of adverse effects.

\begin{abstract}
Artículo: Assessment of the Safety and Efficacy of a New Treatment Strategy with Percutaneous Coronary Intervention (ASSENT-4 PCI) investigators. Primary versus tenecteplase-facilitated percutaneous coronary intervention in patients with ST-segment elevation acute myocardial infarction (ASSENT-4 PCI): randomised trial. Lancet. 2006;18;367:569-78.
\end{abstract}

Antecedentes: una revisión sistemática de 22 ensayos clínicos ${ }^{1}$ mostró que en los enfermos con síndrome coronario agudo con elevación del segmento ST (SCACEST) la angioplastia primaria, en comparación con el tratamiento fibrinolítico reduce la mortalidad y el reinfarto no fatal precoces (6 semanas) del 7,4\% al $5,3 \%$, y del $6,8 \%$ al $2,5 \%$, respectivamente. Las guías de práctica clínica, tanto en Europa como en EE.UU. ${ }^{2,3}$, recomiendan el empleo de la intervención coronaria percutánea como tratamiento de elección del SCACEST cuando sea posible realizarla en los 90 minutos siguientes al comienzo de los síntomas, ya que la eficacia de la fibrinolisis es similar a la de la angioplastia primaria cuando el intervalo entre la aparición de los síntomas y cualquiera de los 2 tratamiento se realiza a la 2-3 horas del comienzo de los síntomas.

Sin embargo, el llevar esta recomendación a la práctica clínica es difícil, ya que para la realización de angioplastia coronaria es preciso trasladar al paciente a un centro hospitalario que disponga de laboratorio de hemodinámica.

*Esta sección está financiada con la ayuda ISCIII-FIS 02/8115.
Para este traslado se requiere, en muchas ocasiones, un tiempo superior a las 3 horas. Por tanto es razonable evaluar la eficacia de un tratamiento inicial con fibrinolíticos o inhibidores IIb/IIIa previo a la realización de la angioplastia con el fin de mantener el principio de que en el tratamiento del infarto agudo de miocardio «tiempo es músculo». La angioplastia facilitada, empleo de fibrinolíticos, inhibidores IIb-IIIa, o la combinación de ambos antes de la realización de la angioplastia, también ha sido propuesta tras el hallazgo en estudios previos en los que se mostraron mejores resultados de la angioplastia cuando se realizaba sobre un vaso abierto que cuando se hacía sobre un vaso ocluido.

La angioplastia facilitada ha sido evaluada en ensayos clínicos de pequeño tamaño muestral, que han proporcionado resultados discrepantes.

Objetivo: estimar el efecto sobre un desenlace combinado de muerte, insuficiencia cardíaca o shock a los 90 días de la asignación aleatoria, del empleo de fibrinólisis con tenecteplasa previa a la realización de una intervención percutánea coronaria (angioplastia facilitada) frente a la realización de una angioplastia primaria convencional en pacientes con SCACEST.

Diseño: ensayo clínico con asignación aleatoria. 
Tabla 1: Características generales

\begin{tabular}{|c|c|c|}
\hline & $\begin{array}{l}\text { Grupo angioplastia facilitada } \\
\qquad(\mathrm{n}=829)\end{array}$ & $\begin{array}{c}\text { Grupo angioplastia primaria } \\
\qquad(\mathrm{n}=838)\end{array}$ \\
\hline Edad, años, media & 61 & 60 \\
\hline Género, mujeres, \% & 23 & 23 \\
\hline Killip I(\%) & 91 & 93 \\
\hline Tensión arterial, mmHg, media & 134 & 134 \\
\hline \multicolumn{3}{|l|}{ Localización del Infarto agudo miocardio, \% } \\
\hline Anterior & 49 & 46 \\
\hline Inferior & 50 & 51 \\
\hline IAM previo, \% & 13 & 11 \\
\hline Angioplastia previa, \% & 9 & 8 \\
\hline Cirugía coronaria previa, \% & 2 & 2 \\
\hline \multicolumn{3}{|l|}{ Patología previa, \% } \\
\hline Hipertensión arterial & 47 & 47 \\
\hline Diabetes & 17 & 16 \\
\hline \multicolumn{3}{|l|}{ Tiempo entre comienzo de sintomatología } \\
\hline y la asignación aleatoria, minutos, mediana & 140 & 135 \\
\hline \multicolumn{3}{|l|}{ Tiempo entre comienzo de sintomatología } \\
\hline y tenecteplasa, minutos, mediana & 153 & - \\
\hline \multicolumn{3}{|l|}{ Tiempo entre comienzo de sintomatología } \\
\hline y balón, minutos, mediana & 263 & 255 \\
\hline Tiempo entre aleatorización y balón (minutos) (mediana) & 115 & 107 \\
\hline \multicolumn{3}{|l|}{ Flujo TIMI antes de angioplastia, \% } \\
\hline 0 & 24 & 62 \\
\hline 1 & 10 & 9 \\
\hline 2 & 21 & 13 \\
\hline 3 & 43 & 15 \\
\hline Angioplastia sobre vaso responsable, \% & 87 & 93 \\
\hline Colocación de stent, \% & 80 & 85 \\
\hline Flujo TIMI 3 tras la angioplastia, \% & 88 & 89 \\
\hline Inhibidores de la glicoproteína Ila/IIlb, \% & 13 & 50 \\
\hline
\end{tabular}

IAM: infarto agudo de miocardio

Enmascarado: no.

Los desenlaces principales fueron asignados por un comité que desconocía el tratamiento asignado a cada paciente, de acuerdo con unos criterios predefinidos.

Lugar: veinticuatro países.

En tres localizaciones: 1 . Hospitales de nivel terciario (45\% de los pacientes); 2. Hospitales sin posibilidad de realización de intervencionismo coronario in situ $(35 \%)$ y 3. ambulancias en las que es posible la fibrinólisis prehospitalaria (20\%).

Período de estudio: entre noviembre de 2003 y abril de 2005.

Pacientes (tabla 1 y figura 1): Criterios de Inclusión: todos los siguientes: 1. Edad $\geq 18$ años. 2. Comienzo de los síntomas $\leq 6$ horas hasta la asignación aleatoria. 3. Intención de llevar a cabo una angioplastia primaria. 4. Elevación del segmento $\mathrm{ST} \geq 0,6$ $\mathrm{mV}$ en múltiples derivaciones, o en caso de infarto inferior elevación de $\mathrm{ST} \geq 0,4 \mathrm{mV}$ en II, III y aVF, o aparición de un bloqueo completo de rama izquierda con elevación concordante de $\mathrm{ST} \geq 0,1 \mathrm{mV}$.
Criterios de exclusión: 1 . Tiempo estimado a la realización del cateterismo inferior a 1 hora o superior a las 3 horas tras la asignación aleatoria. 2. Dificultad prevista para el acceso vascular. 3. Participación previa en el ensayo clínico. 4. Contraindicaciones habituales para el tratamiento fibrinolítico ${ }^{4}$.

\section{Intervenciones que se comparan:}

Grupo angioplastia facilitada: realización de un procedimiento percutáneo invasivo precedido de la administración de una dosis ajustada al peso de tenecteplasa: peso < $60 \mathrm{~kg}$; $30 \mathrm{mg}$; peso entre 60 y $69 \mathrm{~kg}, 35 \mathrm{mg}$, peso 70 a $79 \mathrm{~kg}$, $40 \mathrm{mg}$; peso entre 80 y $89 \mathrm{~kg}, 45 \mathrm{mg}$; peso $\geq 90 \mathrm{~kg}, 50 \mathrm{mg}$.

En este grupo no estaba permitido el empleo de inhibidores IIb-IIIa salvo que se objetivara un gran trombo residual no recanalizado adecuadamente en la arteria responsable del infarto agudo de miocardio.

Grupo angioplastia primaria: realización de procedimiento percutáneo invasivo estándar. En este grupo el empleo de inhibidores IIb-IIIa era decidido por el investigador.

Todos los pacientes recibieron: 1 . Una dosis de ácido acetilsalicílico entre 150 y 325 mg. 
Figura 1: Diagrama de inclusión

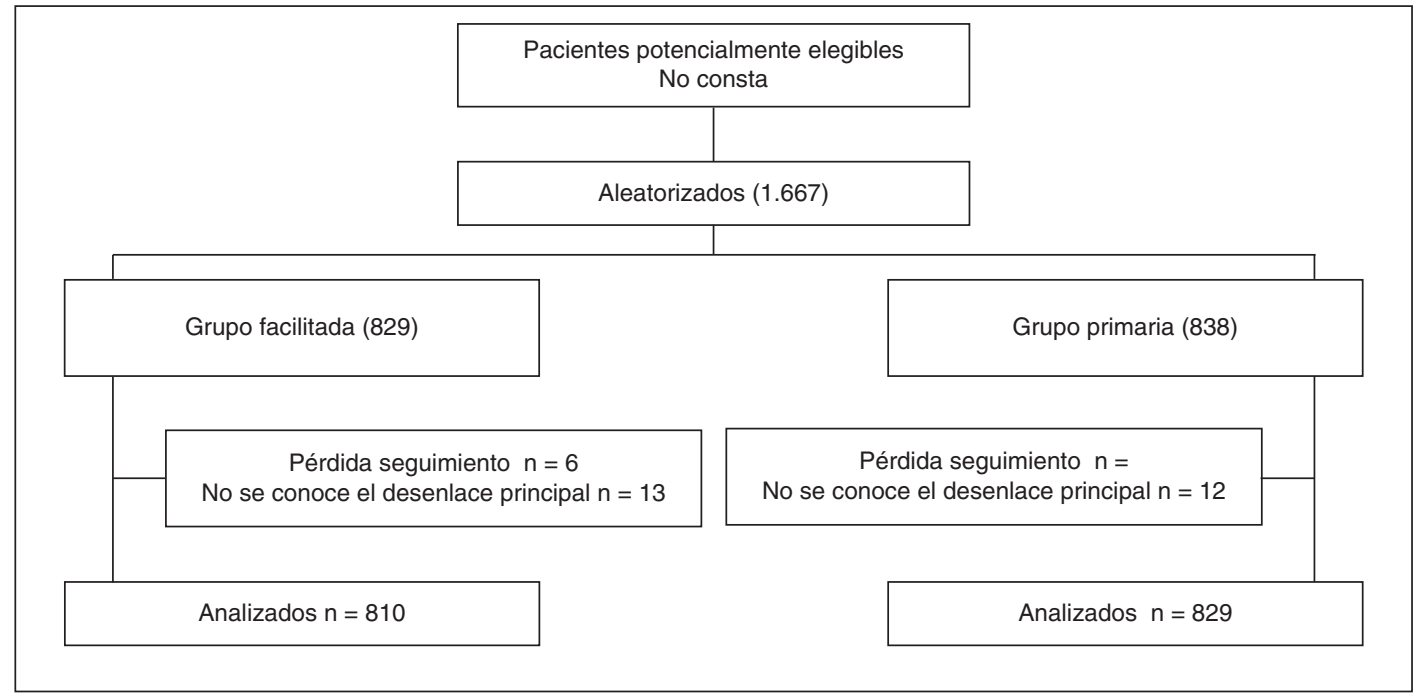

Tabla 2: Desenlace combinado de muerte, insuficiencia cardíaca o shock a los 90 días del tratamiento

\begin{tabular}{|lc|}
\hline Grupo angioplastia facilitada & $151 / 810(18,6 \%)$ \\
Grupo angioplastia primaria & $110 / 819(13,4 \%)$ \\
RR (IC 95\%) & $1,39(1,11$ a 1,74$)$ \\
IRR (IC 95\%) & $38,8 \%(10,8 \%$ a $73,9 \%)$ \\
IAR (IC 95\%) & $5,2 \%(1,7 \%$ a $8,8 \%)$ \\
NNP (IC 95\%) & $20(112$ a 59$)$ \\
\hline
\end{tabular}

La mortalidad a los 90 días fue $7 \%$ en el grupo de angioplastia facilitada y $5 \%$ en el grupo de angioplastia primaria $(\mathrm{p}=0,14)$, la incidencia de insuficiencia cardíaca $12 \%$ y $9 \%$ respectivamente $(\mathrm{p}=0,06)$; la incidencia de shock $6 \%$ y $5 \%(\mathrm{p}=0,19)$, respectivamente.

RR: riesgo relativo; IAR: incremento absoluto riesgo; IRR: incremento relativo riesgo; NNP: número necesario para perjudicar IC: intervalos de confianza.

2. Un bolo de heparina no fraccionada. En el grupo de angioplastia facilitada $60 \mathrm{U} / \mathrm{kg}$ con un máximo de 4000 U. En el grupo de angioplastia primaria $70 \mathrm{U} / \mathrm{kg}$. No se administró en ningún caso perfusión continua de heparina, aunque se empleó un segundo bolo de heparina durante la intervención para conseguir un tiempo de coagulación activado de 350-400 sec (250-300 sec si se empleaban inhibidores IIb-IIIa) en el grupo angioplastia primaria y de 300-350 sec en el grupo angioplastia facilitada.

El procedimiento a realizar sobre la arteria afectada era decidido por el médico responsable. En caso de colocación de stent se administró una dosis de carga de clopidogrel de $300 \mathrm{mg}$ y luego una dosis de mantenimiento de $75 \mathrm{mg} / 24$ horas.

Desenlaces principales: desenlace combinado de muerte, insuficiencia cardíaca o shock a los 90 días de la asignación aleatoria.

Análisis por intención de tratar: sí.

Resultados principales (tablas 2 y 3 ): el estudio se interrumpió tras alcanzar la mitad del tamaño muestral prefijado, al observarse un aumento de la mortalidad hospitalaria en el grupo de angioplastia facilitada. El análisis por los subgrupos predefinidos en protocolo del estudio mostró la consistencia de los hallazgos que fueron homogéneos en todos esos subgrupos.

Información sobre costes: no consta.

Financiación del estudio: Boehringer-Ingelheim y Genentech.

Conflicto de intereses: está adecuadamente reflejado en el texto.

Tabla 3: Complicaciones hemorrágicas

\begin{tabular}{|l|c|c|}
\hline & $\begin{array}{c}\text { Grupo angioplastia facilitada } \\
(\mathrm{n}=829)\end{array}$ & $\begin{array}{c}\text { Grupo angioplastia primaria } \\
(\mathrm{n}=838)\end{array}$ \\
\hline Ictus en el hospital, \% & 2 & - \\
Complicación hemorrágica mayor, \% & 6 & 4 \\
Transfusión sanguínea, \% & 6 & 4 \\
\hline
\end{tabular}


Conclusiones de los autores: el empleo de una dosis estándar de tenecteplasa previo a la realización de un angioplastia en las siguientes horas (entre 1 y 3 horas) se asocia con una mayor tasa de complicaciones que la realización de una angioplastia convencional.

Conclusiones de los revisores: el principal hallazgo de este estudio es que la angioplastia facilitada con tenecteplasa se asocia a una mayor incidencia de efectos adversos, cardíacos y extracardíacos, a pesar de que alcanza un mayor grado de permeabilidad de la arteria responsable del infarto, flujo TIMI $345 \%$ frente al 15\%, antes de realizar la intervención percutánea. Con independencia de que los mecanismos fisiopatológicosque puedan explicar estos hallazgos, y que los autores discuten en el texto, se pueden obtener dos conclusiones. En primer lugar la estrategia de angioplastia facilitada evaluada en este ensayo no debe trasladarse a la práctica clínica. En segundo lugar la permeabilidad coronaria estimada por el flujo TIMI no es un buen estimador (subrogado) de la utilidad clínica de la angioplastia facilitada.

Los resultados de este ensayo clínico son congruentes con los de un metaanálisis ${ }^{5}$ que ha revisado 17 ensayos clínicos, incluido éste, con 4.504 pacientes, que han comparado diferentes estrategias de angioplastia facilitada con angioplastia primaria. En este metaanálisis la angioplastia facilitada, en comparación con la angioplastia primaria, se asocia a: $a$ ) mayor porcentaje de enfermos con flujo TIMI 3 antes de realizar la angioplastia, $37 \%$ vs $15 \%$; $b$ ) mayor mortalidad, $5 \%$ frente al $3 \%$; c) mayor incidencia de reinfarto no fatal, $3 \%$ frente al $2 \%$; d) mayor necesidad de revascularización urgente, $4 \%$ frente al $1 \% ; e$ ) mayor riesgo de sangrado grave, $7 \%$ frente al $5 \%$.

Estos hallazgos muestran que no existe actualmente soporte para implementar ninguna estrategia de angioplastia facilitada evaluada hasta ahora. Por lo que en el futuro será necesario comparar nuevos tratamientos con nuevas combinaciones de fármacos - fibrinolíticos, antiagregantes y antitrombina - y nuevos intervalos de tiempos ajustados a la práctica habitual de la atención al enfermo coronario - desde aparición de síntomas hasta la atención, traslado a centros con hemodinámica ${ }^{6}$.

\section{Bibliografía}

1. Keeley EC, Boura JA, Grines CL. Comparison of primary and facilitated percutaneous coronary interventions for ST-elevation myocardial infarction: quantitative review of randomised trials. Lancet 2003;361:13-20. (Revisado Med Intensiva. 2003;27:632-4).

2. Van de Werf F, Ardissino D, Betriu A, Cokkinos DV, Falk E, Fox KA, et al Task Force on the Management of Acute Myocardial Infarction of the European Society of Cardiology. Management of acute myocardial infarction in patients presenting with ST-segment elevation. The Task Force on the Management of Acute Myocardial Infarction of the European Society of Cardiology. Eur Heart J. 2003;24:28-66.

3. Antman EM, Anbe DT, Armstrong PW, Bates ER, Green LA, Hand M, et al. ACC/AHA guidelines for the management of patients with ST-elevation myocardial infarction; A report of the American College of Cardiology/American Heart Association Task Force on Practice Guidelines (Committee to Revise the
1999 Guidelines for the Management of patients with acute myocardial infarction). J Am Coll Cardiol. 2004;44:e1-e211.

4. Assessment of the Safety and Efficacy of a New Thrombolytic Regimen (ASSENT)-3 Investigators. Efficacy and safety of tenecteplase in combination with enoxaparin, abciximab, or unfractionated heparin: the ASSENT-3 randomised trial in acute myocardial infarction. Lancet. 2001;358:605-13.

5. Keeley EC, Boura JA, Grines CL. Comparison of primary and facilitated percutaneous coronary interventions for ST-elevation myocardial infarction: quantitative review of randomised trials. Lancet. 2006;367:579-88.

6. Ellis SG, Armstrong P, Betriu A, Brodie B, Herrmann H, Montalescot G, et al. Facilitated Intervention with Enhanced Reperfusion Speed to Stop Events Investigators.Facilitated percutaneous coronary intervention versus primary percutaneous coronary intervention: design and rationale of the Facilitated Intervention with Enhanced Reperfusion Speed to Stop Events (FINESSE) trial. Am Heart J. 2004;147:e16. 